\title{
The use of secondments as a tool to increase knowledge translation
}

Lily O’Donoughue Jenkins ${ }^{a, b}$ and Kaarin J Anstey ${ }^{a}$

${ }^{a}$ Centre for Research on Ageing, Health and Wellbeing, The Australian National University, Canberra, ACT

b Corresponding author: lily.jenkins@anu.edu.au

\section{Article history}

Publication date: February 2017

O'Donoughue Jenkins L, Anstey KJ. The use of secondments as a tool to increase knowledge translation. Public Health Res Pract. 2017;27(1):e2711708. doi: http:// dx.doi.org/10.17061/phrp2711708

\section{Key points}

- Secondments have received very little attention in the knowledge translation (KT) literature

- This study looks at a reciprocal secondment as an example of how secondments could be used as a tool to increase KT by developing individual capacity

- Recommendations based on the case studies provided include creating a formal link between government and research organisations, trialling different types of secondments, and having a detailed evaluation plan to measure the success of the secondment

\section{Abstract}

This paper discusses the use of secondments as a tool to increase knowledge translation between academics and policy makers by developing individual capacity. A case study is presented of a reciprocal secondment between a government department and a university. Enablers of knowledge translation included flexibility and support, a prior relationship between the two organisations, and a government culture that values use of research in policy making. Barriers included the lack of a planned approach with agreed outcomes, and a lack of evaluation at the end of the secondment. Recommendations for future secondments include establishing ongoing secondments between organisations; trialling different types of secondments; and having a detailed plan at the beginning of a secondment, including how the success of the secondment will be measured, and a formal evaluation at the end.

\section{Introduction}

Knowledge translation (KT) is defined by the Canadian Institute of Health Research as a dynamic and iterative process that includes synthesis, dissemination, exchange and ethically sound application of knowledge to improve health, provide more effective health services and products, and strengthen the healthcare system. ${ }^{1}$ There are three models of KT: the 'push' model emphasises researchers producing and communicating information to potential users ${ }^{2}$; the 'pull' model stresses user-driven research'; and the 'exchange' model emphasises human interaction, with researchers and knowledge users coproducing research and disseminating results. ${ }^{1,3}$

One of the most widely cited barriers to, and enablers of, $\mathrm{KT}$ is the relationship between researchers and policy makers. ${ }^{4}$ To ensure evidence based policy, the interface between research and policy needs to improve. Actively building and maintaining relationships through discussions, meetings or workshops can lead to policy makers contributing to the research process and can increase the likelihood that research findings will inform policy decisions. ${ }^{5}$ One approach to increasing the interaction or improving the interface between researchers and policy makers is use of secondments. ${ }^{6,7,8}$

Secondments have been used in the healthcare setting as a way to bring an individual's expertise to a team, and to develop staff knowledge and skills. ${ }^{9,10}$ In the KT literature, secondments have received very little 
attention. Most of the initiatives used to develop KT research and activity involve courses and educational resources. However, in the experiential learning model, secondments, mentoring and collaborative projects are used to build KT capacity. ${ }^{9}$

Previous research has found that secondments involving researchers and healthcare practitioners are an efficient way to transfer research. ${ }^{11}$ They give practitioners an opportunity to develop knowledge of research processes, and enable researchers to gain a better understanding of what research is relevant to practitioners and how to communicate their findings. ${ }^{7,12}$ Secondments can benefit all parties at a relatively low cost. ${ }^{12,13}$ The secondee has a chance to develop expertise in a specific area, and the host organisation receives some assistance and an external perspective on its projects. The seconding organisation also benefits from skills acquired during the secondment that can be applied to the secondee's substantive position and spread across the organisation to improve the skills of other staff. 9,12,14

Although secondments occur between government departments and academic institutions, there has been minimal research on how they may contribute to increasing KT. ${ }^{12}$ The aim of this paper is to discuss how secondments could be used to instigate and maintain relationships between government departments and universities, and how they could be an effective tool for KT by building individual capacity. This paper shares the experience of two example secondments; discusses the enablers of, and barriers to, these secondments; and provides recommendations for the future.

\section{Reciprocal secondment}

The reciprocal secondment occurred between the Australian National University (ANU) and the Australian Capital Territory (ACT) Health Department (ACT Health). A university researcher (the lead author) spent 10 months part time in a section of ACT Health. The researcher worked on a number of reports and projects within the department, but the secondment did not have a predetermined aim to produce a specific output. A staff member from ACT Health spent approximately 30 working days at the ANU centre that the researcher is from, during a period of 6 months. The secondment to the ANU had a specific project: to prepare a report using a dataset developed by the ANU centre.

Both secondees answered 10 open-ended questions in paper form, developed by the authors, about their experience of the secondment. The questions focused on four main topics: the experience of undertaking the secondment; enablers of, and barriers to, the success of the secondment; the perceived degree of success, and the basis on which these judgements were made; and secondments as a tool for KT.

\section{Discussion}

\section{Secondment experience}

Both secondees found that the secondment was a useful tool for $\mathrm{KT}$, and that it strengthened the relationship between ACT Health and the ANU centre. They found that collaboration between the two organisations increased, and relevant knowledge and skills were exchanged. Through the secondment, the researcher learnt about current policy priorities and real-world constraints, how evidence was accessed and used in ACT Health, and the differences in writing style and use of language between researchers and government employees. This was important for the researcher in gaining a better understanding of how to communicate research findings.

The staff member from ACT Health believed that one of the benefits of the secondment was becoming more aware of academic resources. She found that a high level of support and communication during the secondment from senior staff at both organisations contributed to the success of the project; the two organisations worked collaboratively to develop and refine the project's aim, and the project output was delivered in a timely and high-quality fashion. The secondee recognised how secondments could be used to strengthen $\mathrm{KT}$, stating that:

Secondments enable staff from both organisations

to walk in the shoes of the other and thus promote better understanding of how each one approaches and uses knowledge in the context of their own organisation. Through this process, both organisations learn to share information in a manner that better ensures that the information can be used and understood in a meaningful and relevant way.

\section{Barriers and enablers}

The secondees differed in the amount of time spent at their host organisation and in the work they completed during their secondments. During the secondments, the participants undertook two roles concurrently and managed two workloads. In Gerrish and Piercy's study ${ }^{9}$, secondees experienced difficulties in ensuring that sufficient priority was given to both roles. In our study, the secondments were flexible, so the secondees' usual workloads and the amount of time spent at either organisation could be adjusted. Previous research has found that flexibility is important to the success of secondments ${ }^{15}$, so it was beneficial that both organisations supported these adjustments.

In relation to evaluating the success of the secondments, the secondment to the ANU was easier to evaluate because there was a specific output. This output was produced collaboratively, and generated research that ACT Health used to inform current policies 
and programs. By working on a range of projects, the secondee to ACT Health learnt more about government processes and created more links within the organisation. Neither secondment had an agreed evaluation plan before commencement.

Consistent with previous studies, 3,9,12,14, the reciprocal secondment benefited both organisations and increased the secondees' capacity in relevant skills that could facilitate KT. ${ }^{13,15-17}$ The ACT Health staff member increased her knowledge of current research, and developed skills in statistical analyses, and writing and publication of research. The researcher learnt more about the process of policy making, and the structure and culture of the government organisation, including issues facing the organisation. Although secondments develop an individual's capacity, this knowledge can also be translated to the organisation level and maximise KT efforts. ${ }^{17}$ For example, when academic colleagues wanted to discuss their research with staff in ACT Health, the researcher was able to give the information to the appropriate person in the department.

Some aspects facilitated these secondments. Firstly, both secondees were familiar with the seconded organisation through previous experience. As Newman et al. ${ }^{16}$ found, this provides some foundation for the relationship because both parties already have some cultural understanding of, and respect for, the other. Secondly, the current senior leaders in ACT Health are advocating for more research development from staff, including publishing of journal articles and collaboration with academics. This allowed the secondment process to proceed easily and efficiently.

\section{Recommendations for the future}

The first recommendation is concerned with maintaining and building the relationship in future. To prevent decreases in communication between the two organisations and to maintain momentum, Black and Martyn ${ }^{13}$ suggest that a formal, even contractual, link be developed. One way of doing this is creating a partnership between the organisations through regular, perhaps annual, secondments. Secondments could occur between different departments and across all staff levels. A formal link may help to ensure that opportunities for interaction between the two organisations are maintained in the long term. ${ }^{18}$ These collaborations could produce culture shifts, creating a decision-relevant culture among researchers, and a research-attuned culture among decision makers. ${ }^{2}$

The second recommendation is to trial different types of secondments to see which is more successful for KT. For example, organisations could consider short-term secondments with highly focused and defined objectives, or long-term, ongoing secondments. The success of the secondment type depends on the department, the project in question and the people involved. For example, Gerrish and Piercy ${ }^{9}$ found that clinical secondees benefited from ongoing clinical engagement through parttime secondments, but that academic secondees had difficulties managing dual workloads and benefited more from a full-time secondment.

Thirdly, it is recommended that secondments have a detailed plan before commencement and a formal evaluation of how successful the secondment was. For example, in Black and Martyn's secondment ${ }^{13}$, they established a broad agreement covering basic contractual details, accountability, expenses and regular reviews. An evaluation at the end of the secondment could provide evidence of whether the secondment was effective in meeting outcomes, whether it met key objectives, what was gained by doing the secondment and any changes required for future secondments. ${ }^{10}$

Few projects have evaluated and documented the outcomes of KT processes. This may be because of difficulties defining, and thus measuring, a successful outcome. ${ }^{8}$ One way to evaluate the outcomes of a secondment is to look at changes in practice or policy - for example, incorporation of relevant research into a policy. A second is to look at changes in understanding for example, through increased knowledge, and changes in attitude and thinking. Finally, the processes used for KT can be evaluated, including how KT was conducted (e.g. formal arrangements, leadership, communication) and the quality of the processes (e.g. quality of information, cost-effectiveness)..$^{19}$

\section{Conclusion}

By examining two cases, this paper shows that secondments can increase KT between researchers and government departments by developing individual capacity. Barriers to the secondments included the lack of a planned approach with agreed outcomes and a lack of evaluation. Enablers included support and flexibility from both organisations, an existing relationship between the organisations, and a government culture that valued the use of research in decision making. Future research and monitoring are required to demonstrate the long-term outcomes of secondments, including for organisations. These outcomes may include a stronger relationship between the organisations, an increase in shared projects, and increased use of research in the policy process. 


\section{Acknowledgements}

Thanks to Cathy Baker for providing her experience of the secondment to ANU, and to Professor Gabriele Bammer for her comments and editing. LOJ is supported by the Australian Research Council Centre of Excellence in Population Ageing Research (project CE110001029) and the Australian Research Council Linkage Project (project LP120200609). KA is funded by NHMRC Fellowship (\#1102694).

\section{Competing interests}

None declared

\section{Author contributions}

LOJ was responsible for collecting data, and for designing, writing and editing the manuscript. KA arranged the secondment, and contributed to the design and editing of the manuscript.

\section{References}

1. Graham ID, Logan J, Harrison MB, Straus SE, Tetroe J, Caswell W, et al. Lost in knowledge translation: time for a map? J Contin Educ Health Prof. 2006;26(1):13-24.

2. Lavis JN, Robertson D, Woodside JM, McLeod CB, Abelson J, Knowledge Transfer Study Group. How can research organizations more effectively transfer research knowledge to decision makers? Milbank $Q$. 2003;81(2):221-48.

3. Bullock H, Watson A, Goering P. Building for success: mental health research with an integrated knowledge translation approach. Can J Commun Ment Health. 2010;29(S5):9-21.

4. Innvaer S, Vist G, Trommald M, Oxman A. Health policy-makers' perceptions of their use of evidence: a systematic review. J Health Serv Res Policy. 2002;7:23944.

5. Dwan KM, Mclnnes PC. Increasing the influence of one's research on policy. Aust Health Rev. 2013;37(2):194-8.

6. Gibbons P, Zammit C, Youngentob K, Possingham HP, Lindenmayer DB, Bekessy S, et al. Some practical suggestions for improving engagement between researchers and policy-makers in natural resource management. Ecolo Manage Restor. 2008;9(3):182-6.

7. Ward V, Smith S, Foy R, House A, Hamer S. Planning for knowledge translation: a researcher's guide. Evid Policy. 2010;6(4):527-41.
8. Cvitanovic C, Hobday AJ, van Kerkhoff L, Wilson SK, Dobbs K, Marshall NA. Improving knowledge exchange among scientists and decision-makers to facilitate the adaptive governance of marine resources: a review of knowledge and research needs. Ocean Coast Manage. 2015;112:25-35.

9. Gerrish K, Piercy H. Capacity development for knowledge translation: evaluation of an experiential approach through secondment opportunities. Worldviews Evid Based Nurs. 2014;11(3):209-16.

10. Dryden H, Rice AM. Using guidelines to support secondment: a personal experience. J Nurs Manag. 2008;16(1):65-71.

11. Lomas J. Using 'linkage and exchange' to move research into policy at a Canadian foundation. Health Aff (Millwood). 2000;19(3):236-40.

12. Bullock A, Morris ZS, Atwell C. Exchanging knowledge through healthcare manager placements in research teams. Service Industries Journal. 2013;33(13-14):136380.

13. Black D, Martyn P. Reciprocal secondment. Nurs Stand. 1999;13(44):42-3.

14. Hamilton J, Wilkie C. An appraisal of the use of secondment within a large teaching hospital. J Nurs Manag. 2001;9(6):315-20.

15. Bullock A, Morris Z, Atwell C. A formative evaluation of the service delivery and organisation (SDO) management fellowships. Cardiff: NIHR Service Delivery and Organisation Programme, 2012 [cited 2016 Nov 3]. Available from: http://socialwelfare. bl.uk/subject-areas/services-activity/health-services/ nihrhealthservicesanddeliveryresearchprogramme/ 1539182012_SDO_FR_09-1003-01_V01.pdf

16. Newman L, Biedrzycki K, Patterson J, Baum F Partnership in knowledge creation: lessons learned from a researcher-policy actor partnership to co-produce a rapid appraisal case study of South Australia's Social Inclusion Initiative. Evid Policy. 2011;7(1):77-96.

17. Kislov R, Waterman H, Harvey G, Boaden R. Rethinking capacity building for knowledge mobilisation: developing multilevel capabilities in healthcare organisations. Implement Sci. 2014;9:166.

18. Conklin J, Stolee P, Luesby D, Sharratt M, Chambers LW. Enhancing service delivery capacity through knowledge exchange: The Seniors Health Research Transfer Network. Healthc Manage Forum. 2007;20(4):20-26.

19. Fazey I, Bunse L, Msika J, Pinke M, Preedy K, Evely AC, et al. Evaluating knowledge exchange in interdisciplinary and multi-stakeholder research. Global Environ Chang. 2014;25:204-20.

\section{Copyright: (c) (i) (-)}

(C) 2017 O'Donoughue Jenkins and Anstey. This article is licensed under the Creative Commons Attribution-NonCommercial-ShareAlike 4.0 International Licence, which allows others to redistribute, adapt and share this work non-commercially provided they attribute the work and any adapted version of it is distributed under the same Creative Commons licence terms. See: www.creativecommons.org/licenses/by-nc-sa/4.0/ 